\title{
Does the Platelet Index Have a Guiding Role in the Association of Cancer and Pulmonary Thromboembolism?
}

\author{
Ramazan Ocal ${ }^{1}$, Nesrin Ocal, , * \\ ${ }^{1}$ Health Sciences University, Gulhane Medical Faculty, Department of Hema- \\ tology, Ankara, Turkey \\ ${ }^{2}$ Health Sciences University, Gulhane Medical Faculty, Department of Chest \\ Diseases, Ankara, Turkey \\ *Corresponding author: Nesrin Ocal, Health Sciences University, Gulhane Medical \\ Faculty, Department of Chest Diseases, Ankara, Turkey. Tel: +903123044416; Fax: \\ +903123044407; E-mail: nesrinbaygin@yahoo.com
}

DOI:10.30699/mci.4.2.6

Submitted: 3 February 2020

Revised: 27 February 2020

Accepted: 16 March 2020

e-Published: 1 April 2020

Keywords:

Neoplasms

Pulmonary Embolism

Blood Platelets

Blood Cell Count

Mean Platelet Volume

Platelet Count
Introduction: The diagnostic value of the D-dimer test varies with variable platelet numbers and functions in patients suffering from cancer and concomitant pulmonary thromboembolism (PTE). This requires easy and reliable evaluation tests. In this study, we aimed to investigate the hypothesis that platelet functions may be more guiding in the prediction and diagnosis of PTE rather than the number of platelets in cancer patients.

Methods: The clinical, laboratory and radiological findings of all patients diagnosed with PTE were retrieved, retrospectively. Comparisons were performed between "cancer-free" and "cancer" patients. The patients were also evaluated according to their active and remission status.

Results: The data of 232 patients with PTE were reviewed. 172 patients were cancerfree and 60 were diagnosed with cancer. The mean values of D-dimer, mean platelet volume (MPV) and platelet distribution width (PDW) were found to be significantly higher in patients with cancer $(\mathrm{P}=0.015, \mathrm{P}=0.026$, and $\mathrm{P}=0.023$; respectively). On the other hand, mean platelet counts were significantly higher in patients without cancer $(\mathrm{P}=0.05)$. It was also observed that the mean values of $\mathrm{D}$-dimer, Troponin-I, MPV, and $\mathrm{PDW}$ were found to be significantly higher in patients with active cancer $(\mathrm{P}=0.05$, $\mathrm{P}=0.044, \mathrm{P}=0.05$, and $\mathrm{P}=0.042$; respectively). However, the mean platelet counts were significantly higher in patients with cancer who were in remission $(\mathrm{P}=0.05)$.

Conclusions: The morphological characteristics of the platelets are more determinant than the number of platelets in cancer patients with PTE. Platelet indexes such as MPV and PDW may be useful in the prediction, diagnosis, and follow-up of PTE in patients with cancer.

\section{INTRODUCTION}

There is an increased risk of pulmonary thromboembolism (PTE) in patients with malignancy. Moreover, PTE may be the first sign of malignancy which is associated with a high rate of morbidity and mortality in cancer patients. Cancer patients have a known propensity for thrombosis and $20-30 \%$ of all PTEs are cancer-related. PTE is the second cause of death in cancer patients after infections. This tendency to thrombosis in cancer patients may be the result of changes in the amount and activity of the clotting factor or due to the underlying disease itself, treatment of the disease, and changes in platelet function. Also, new evidence suggests that interactions between platelets and 
malignant cells lead to platelet activation and an increased incidence of thrombosis. Thrombotic effects of platelets in cancer patients has become more important; considering both the important roles of platelets in thrombosis and their prognostic role in malignancies [1-4]. The prognostic value of elevated D-dimer in progressive metastatic cancers without thrombosis has been shown in the previous studies [5, 6]. Fibrin formation and its removal occur steadily during cancer progression. On the other hand, thrombotic disorders may range from asymptomatic laboratory changes to massive PTE in cancer patients $[5,6]$. Even in the literature, a case of occult metastatic colorectal adenocarcinoma has been reported by investigating the etiology of D-dimer elevation [7]. On the other hand, the possibility of PTE occurrence in cancer patients with normal D-dimer levels is under investigation. In a large study of active cancer patients, D-dimer levels were not elevated in approximately $3 \%$ of those diagnosed with PTE $[6,8]$. This situation significantly decreases the diagnostic value of high or low D-dimer levels in cancer patients.

Platelets are small blood cells that play a leading role in coagulation and homeostasis. They are circulating anucleate disc-shaped cells with an average half-life of 8-12 days. The normal concentration of platelets is between 150,000 and $400,000 / \mathrm{L}$ in peripheral blood. It is well known that platelets are responsible for the initiation of the hemostatic mechanisms; repairing the injuries made to the vascular endothelium. Platelets act a major role in almost all four major steps of the first phase of hemostasis which is referred to as primary hemostasis. These steps include adhesion to the injury site, activation/secretion, aggregation, and interaction with coagulation factors. In light of these facts, it is obvious that changes in coagulation are one of the key points in thrombosis and thus in the pathogenesis of thromboembolism. This is the main basis of the close relationship between platelets and PTE $[9,10]$. The qualitative and quantitative properties of platelets have been shown to play an important role in the pathogenesis, morbidity, and mortality of patients with PTE. Platelet volume indices are simple and low-cost tests that are measured as part of complete blood counts (CBC) and are easy ways to assess platelet functions. Circulating platelets differ in size and hemostatic potential. The mean platelet volume (MPV), indicative of this variability, is usually routinely studied as part of a CBC. MPV is determined during both megakaryopoiesis and thrombopoiesis. MPV is a simple and accurate sign of platelet activation and platelet function $[11,12]$. Platelet distribution width (PDW) has also been demonstrated as another sign of platelet activation which measures the variability in platelet size. Additionally, a significant association between increased MPV and both arterial and venous diseases such as myocardial infarction (MI) and PTE, has been shown in previous studies [13, 14].

There is a need for an easy and quick assessment method for screening PTE in cancer patients. Considering both the suspected diagnostic value of D-dimer in cancer patients and the significant role of platelet index in PTE, the idea has emerged that this relationship may play a prognostic role in the evaluation of PTE in cancer patients. In this study, we aimed to investigate the hypothesis that platelet functions may be more guiding in the prediction and diagnosis of PTE rather than the number of platelets in cancer patients.

\section{METHODS}

Initially, the approval was obtained from the ethics committee of the faculty for this retrospective study. Subsequently, the patients who were diagnosed with acute PTE between the dates October 2016-October 2019 in Health Sciences University, Gulhane Medical Faculty, Chest Diseases Department (Ankara, Turkey) were evaluated, retrospectively. Patients who had been diagnosed in another health center, whose data on the date of diagnosis could not be reached or missing and patients who were out of the follow-up were excluded from the study. Additionally, the patients with thrombocytopenia due to medical treatments or chronic conditions and patients with active hematological diseases were excluded from the study. All patients' demographic data, hemogram results, Well's score, pulmonary embolism severity index (PESI), and simplified version of PESI (SPESI), mortality risk scores, malignancy status, type of malignancy (if any), hospitalization period, and mortality status were recorded. Other indices in the $\mathrm{CBC}$ including hemoglobin (HGB), hematocrit (HCT), white blood cell (WBC), platelet (PLT), PDW, and MPV were 
retrieved. Comparisons were performed between "cancer-free" and "cancer" patients. The patients were evaluated according to their active and remission status. Malignancy types were also examined. The statistical relationships between investigated parameters were evaluated. The obtained data were analyzed by SPSS for Mac 20.0 package program (SPSS Inc, Chicago, IL, USA). The meanपstandard deviation (SD) for all variables were calculated. The mean 7 SD for continuous variables, frequency, and percentage for discrete data were used in descriptive statistics. SDs were determined with the mean values for each parameter. For the analysis of demographic data, groups and subgroups values (\%) were calculated with numerical data. The normality of the continuous variables was analyzed with the Kolmogorov-Smirnov test. In the assessment of correlations, the Spearman test was used for data with non-parametric distribution; while Pearson test was applied for data with the parametric distribution. Mean values with non-parametric distributions were compared by Mann-Whitney U and Kruskal-Wallis $\mathrm{H}$ tests. Mean values with parametric distribution between groups were compared by Student's T test and Oneway Analysis of Variance (ANOVA). P-value less than 0.05 was considered as statistically significant.

\section{RESULTS}

In the last 3 years, 312 patients with PTE were followed up in our clinic. Data from 232 patients were eligible for inclusion in the study. A total of 172 patients were cancer-free and 60 were affected with cancer. The data of 232 patients with PTE were reviewed, retrospectively. A total of 120 patients were female and 112 were male. The mean age of the patients was 66.48 years. Previous deep vein thrombosis (DVT) / PTE history was present in $16.38 \%$ of the cases. Acute DVT was positive in $36.21 \%$. The mean hospital stay was 7.02 days. When the early mortality risks of the patients were evaluated, it was observed that 21 cases $(9.05 \%)$ had high mortality risk (massive), 63 cases $(27.15 \%)$ had intermediate-high mortality risk, 83 cases $(35.77 \%)$ had intermediate-low mortality risk, and 75 cases $(32.32 \%)$ had low mortality risk. Comparison of cancer-free and cancer patients: When the general clinical features were examined, there was no significant difference in the age and gender distribution of these two groups. Previous DVT or PTE history did not differ between the groups but acute DVT was significantly more frequent in cancer patients $(\mathrm{P}=0.05)$. While the mean of Well's and PESI scores were significantly higher in cancer patients, there was no significant difference in the mean sPESI score $(\mathrm{P}=0.045, \mathrm{P}=0.042$, and $\mathrm{P}=0.7$; respectively). When the first 30 -day early mortality risks were examined, it was seen that the intermediate-high and intermediate-low risk groups were more frequent in cancer patients and the low mortality risk was more frequent in cancerfree patients $(\mathrm{P}=0.034, \mathrm{P}=0.041$, and $\mathrm{P}=0.038$; respectively). High mortality risk did not differ between the groups. Similarly, the early mortality of patients did not differ significantly. Similar results were found during the hospitalization (Table 1). When the laboratory values were examined, the mean values of D-dimer, MPV, and PDW were found to be significantly higher in patients with cancer ( $\mathrm{P}=0.015, \mathrm{P}=0.026$, and $\mathrm{P}=0.023$; respectively). On the other hand, the mean platelet counts were significantly higher in patients without cancer $(\mathrm{P}=0.05)$. Other laboratory parameters did not show a significant difference between groups (Table 1). The localization of thrombi causing PTE was also investigated. In general, the settlements dominating the table were recorded. The presence of thrombus in the pulmonary artery trunk and the main pulmonary arteries was more frequent in patients with cancer $(\mathrm{P}=0.043$ and $\mathrm{P}=0.05$; respectively). Other localization did not differ significantly between groups (Table 2).

\section{Evaluation of Cancer Patients}

A total of 47 patients had active cancer and 13 individuals were in remission. The mean age and gender distributions did not differ significantly between the two groups. When cancer areas were examined, lung, colorectal, breast, and ovarian malignancies were found to be the most common in patients with active cancer (Figure 1). In patients who were in remission period, lung, colorectal and prostate cancer were most common (Figure 2).

Previous DVT and/or PTE, and acute DVT were significantly more frequent in patients with active cancer $(\mathrm{P}=0.012$ and $\mathrm{P}=0.05$; respectively). Well's and PESI scores were not significantly different between the groups, whereas the mean sPESI was 
Ocal et al.

Table 1: Comparison of Cancer-Free and Cancer Patients ${ }^{\mathrm{a}}$

\begin{tabular}{|c|c|c|c|}
\hline & Cancer-Free Patients & Cancer Patients & P Value \\
\hline Cases, No. & 172 & 60 & - \\
\hline Age, yr, mean & 67 & 65 & NS \\
\hline \multicolumn{4}{|l|}{ Gender, No.(\%) } \\
\hline Female & $94(54.65)$ & $26(43.33)$ & NS \\
\hline Male & $78(45.35)$ & $34(56.66)$ & NS \\
\hline Previous DVT/PTE History, No.(\%) & $29(16.86)$ & $9(15.00)$ & NS \\
\hline Acute DVT, No.(\%) & $57(33.14)$ & $27(45.00)$ & 0.050 \\
\hline Well's Score & 4.17 & 5.32 & 0.045 \\
\hline PESI Points & 105.71 & 122.78 & 0.042 \\
\hline sPESI Points & 2.12 & 2.51 & NS \\
\hline \multicolumn{4}{|l|}{ Early Mortality Risk (first 30 days), No.(\%) } \\
\hline High & $16(9.30)$ & $5(8.33)$ & NS \\
\hline Intermediate-High & $39(22.67)$ & $24(40.00)$ & 0.034 \\
\hline Intermediate-Low & $56(32.56)$ & $27(45.00)$ & 0.041 \\
\hline Low & $61(35.46)$ & $14(23.33)$ & 0.038 \\
\hline Length of Hospital Stay, d & 7.01 & 7.03 & NS \\
\hline Thrombolytic Therapy, No.(\%) & $21(12.21)$ & $3(5.00)$ & $\longrightarrow^{\mathrm{b}}$ \\
\hline Early Mortality Rate (first 30 days), No.(\%) & $14(8.14)$ & $7(11.66)$ & NS \\
\hline D-Dimer, ng/mL & 5139.91 & 9172.82 & 0.015 \\
\hline Trop-I, pg/mL & 113.82 & 152.67 & NS \\
\hline CK-MB, U/L & 6.66 & 8.42 & NS \\
\hline Nt-ProBNP & 2504.73 & 2258.38 & NS \\
\hline HGB, g/L & 12.71 & 11.55 & NS \\
\hline НCT, \% & 38.69 & 36.35 & NS \\
\hline WBC $\left(\times 10^{3}\right.$ cell $\left./ \mu \mathrm{L}\right)$ & 10.48 & 11.62 & NS \\
\hline Platelet $\left(\times 10^{3}\right.$ cell $\left./ \mu \mathrm{L}\right)$ & 292883.67 & 214661.76 & 0.050 \\
\hline MPV, fL & 8.21 & 10.01 & 0.026 \\
\hline PDW & 9.91 & 12.31 & 0.023 \\
\hline ESR & 46.45 & 50.25 & NS \\
\hline CRP & 74.67 & 76.31 & NS \\
\hline
\end{tabular}

${ }^{a}$ Abbreviations: CRP, C-reactive protein; DVT, deep vein thrombosis; ESR, erythrocyte sedimentation rate; HCT, hematocrit; HGB, hemoglobin; MPV, mean platelet volume; NS, non-significant; PDW, platelet distribution width; PESI, pulmonary embolism severity index; PTE, pulmonary thromboembolism; sPESI, simplified pulmonary embolism severity index; WBC, white blood cell ${ }^{\mathrm{b}}$ Since the number of thrombolytic cases was low, statistical comparison was not appropriate.

Table 2: Localizations of Thrombi in Pulmonary Arterial Tree ${ }^{\text {a }}$

\begin{tabular}{lccc}
\hline & Cancer-Free Patients & Cancer Patients & P Value \\
\hline Cases, No. & 172 & 60 & - \\
Trunk of PA, No.(\%) & $6(3.5)$ & $5(8.3)$ & 0.043 \\
Right/Left PA, No.(\%) & $45(26.2)$ & $25(41.6)$ & 0.050 \\
Lobar Branches, No.(\%) & $95(55.2)$ & $43(71.6)$ & NS \\
Segment Branches, No.(\%) & $136(79.1)$ & $54(90.0)$ & NS \\
Subsegment Branches, No.(\%) & $97(56.4)$ & $30(50.0)$ & NS \\
\hline
\end{tabular}

${ }^{a}$ Abbreviations: NS, non-significant; PA, pulmonary artery 
Table 3: Comparison of Patients With Active Cancer and Cancer in Remission ${ }^{\mathrm{a}}$

\begin{tabular}{|c|c|c|c|}
\hline & Active Cancer & Cancer in Remission & P Value \\
\hline Cases, No.(\%) & 47 & 13 & - \\
\hline Age, yr, No.(\%) & 65 & 66 & NS \\
\hline \multicolumn{4}{|l|}{ Gender } \\
\hline Female, No.(\%) & $19(40.42)$ & $6(46.15)$ & NS \\
\hline Male, No.(\%) & $28(59.57)$ & $7(53.84)$ & NS \\
\hline Previous DVT/PTE History, No.(\%) & $8(17.02)$ & $1(7.69)$ & 0.012 \\
\hline Acute DVT, No.(\%) & $22(46.81)$ & $5(38.46)$ & 0.050 \\
\hline Well's Score & 5.48 & 4.69 & NS \\
\hline PESI Points & 126.51 & 115.77 & NS \\
\hline sPESI Points & 2.74 & 1.85 & 0.050 \\
\hline \multicolumn{4}{|l|}{ Early Mortality Risk (first 30 days), No.(\%) } \\
\hline High & $5(10.64)$ & $0(0.00)$ & 0.001 \\
\hline Intermediate-High & $18(38.29)$ & $6(46.15)$ & NS \\
\hline Intermediate-Low & $23(48.94)$ & $4(30.77)$ & 0.05 \\
\hline Low & $9(19.15)$ & $3(23.07)$ & NS \\
\hline Length of Hospital Stay, d & 6.91 & 8.08 & NS \\
\hline Thrombolytic Therapy, No.(\%) & $3(6.38)$ & $0(0.00)$ & $\square^{\mathrm{b}}$ \\
\hline Early Mortality Rate (first 30 days), No.(\%) & $6(12.76)$ & $1(7.69)$ & 0.045 \\
\hline D-dimer, ng/mL & 9321.85 & 8634.02 & 0.050 \\
\hline Trop-I, pg/mL & 158.01 & 66.84 & 0.044 \\
\hline CK-MB, U/L & 8.22 & 8.36 & NS \\
\hline HGB, g/L & 11.47 & 11.14 & NS \\
\hline НСТ, \% & 35.27 & 34.55 & NS \\
\hline WBC $\left(\times 10^{3}\right.$ cell $\left./ \mu \mathrm{L}\right)$ & 12.22 & 10.32 & NS \\
\hline Platelet $\left(\times 10^{3}\right.$ cell $\left./ \mu \mathrm{L}\right)$ & 209520.23 & 233251.21 & 0.047 \\
\hline MPV, fL & 10.51 & 8.21 & 0.050 \\
\hline PDW & 12.92 & 10.11 & 0.042 \\
\hline ESR & 52.51 & 46.25 & NS \\
\hline CRP & 82.63 & 53.46 & NS \\
\hline
\end{tabular}

a Abbreviations: CRP, C-reactive protein; DVT, deep vein thrombosis; ESR, erythrocyte sedimentation rate; HCT, hematocrit; HGB, hemoglobin; MPV, mean platelet volume; NS, non-significant; PDW, platelet distribution width; PESI, pulmonary embolism severity index; PTE, pulmonary thromboembolism; sPESI, simplified pulmonary embolism severity index; WBC, white blood cell

${ }^{\mathrm{b}}$ Since the number of thrombolytic cases was low, statistical comparison was not appropriate.

significantly higher in patients with active cancer $(\mathrm{P}=0.05)$. The first 30 -day early mortality risks were also compared between patients with active cancer and those in remission. High mortality risk and intermediate-low mortality risk were significantly more frequent in patients with active cancer $(\mathrm{P}=0.001$ and $\mathrm{P}=0.05$; respectively). Additionally, the early mortality (mortality in the first 30-days) was more frequent in patients with active cancer
$(\mathrm{P}=0.045)$. Other clinical findings did not show a significant difference between groups (Table 3). The laboratory values were also compared between cancer sub-groups. It was observed that the mean values of D-dimer, Troponin-I, MPV, and PDW were found to be significantly higher in patients with active cancer $(\mathrm{P}=0.05, \mathrm{P}=0.044, \mathrm{P}=0.05$, and $\mathrm{P}=0.042$; respectively). However, the mean platelet counts were significantly higher in patients 


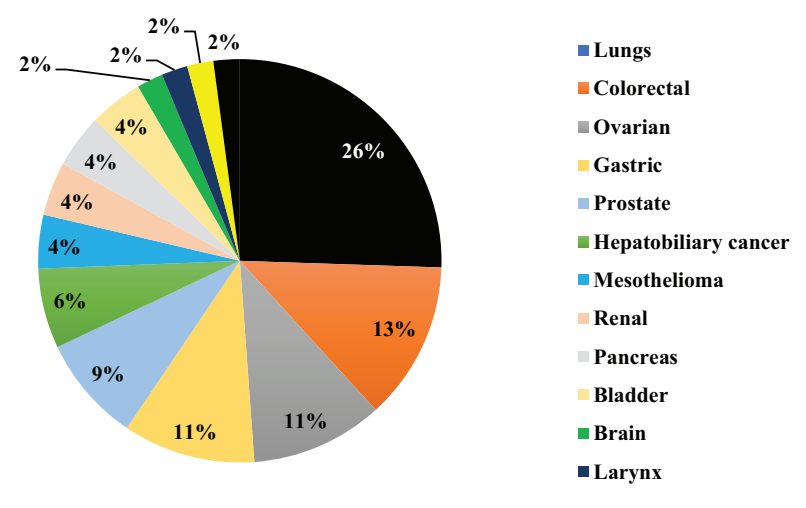

$\overline{\text { Figure 1: Distribution of Cancer Sites in Patients With Active Malignanc }}$

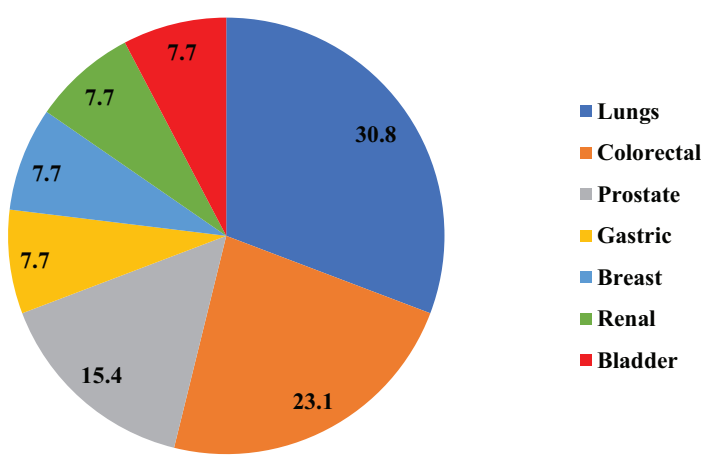

Figure 2: Distribution of Cancer Sites in Patients With Malignancy in Remission

who were in remission $(\mathrm{P}=0.05)$. Other laboratory parameters did not show a significant difference between groups (Table 3).

\section{DISCUSSION}

The correlation between cancer and venous thromboembolism (VTE) was first reported in 1865 by Armand Trousseau [15]. In general, active cancer is considered to increase the risk of PTE by 4-7 times $[16,17]$. So, there is a need for evaluating the modalities that can predict PTE in cancer patients. Although the relationship between cancer and thrombosis has not been fully elucidated, there is sufficient evidence to suggest some potential mechanisms. For example, the association of circulating cancer cells with platelets can increase platelet concentration and facilitate thrombosis. Direct interaction with tumor cells induces platelet aggregation in experimental cancer models. Besides, cancer cells directly excrete thrombin and other mediators, which interact with platelet surface receptors such as P2Y12, PAR-1, PAR-4 and thromboxane receptors. Tumors also secrete matrix metalloproteinases and IL-6, which have been shown to activate platelets directly. Another result of this mechanism other than thrombosis is the increase in the spread of cancer cells $[18,19]$. The second mechanism is the protection of platelets from the cytotoxicity of circulating natural killer cells by wrapping the cancer cells as a protective cape [20, 21]. It has not yet been fully clarified if the number or morphological characteristics of the platelet is more decisive. It is well known that the PTE incidence increases despite the increase in the active platelet count which is frequently observed especially in patients with active cancer undergoing chemotherapy $[2,4,18]$. This suggests that increased thrombocytosis in patients with active cancer is dominated by changes in platelet morphology and function rather than the platelet counts. In light of these findings, evaluation of platelet count alone is not sufficient in cancer patients and PDW and MPV, which are morphological indicators of platelet index, may also be important. In fact, with a retrospective cohort study in which all these patients were monitored for pre-PTE platelet levels, this confusion could be further illuminated. When our results were examined in terms of isolated platelet counts, it was seen that cancer patients, especially patients with active cancer, had lower platelet counts. However, despite the low platelet count, the higher thrombus burden in patients with active cancer supports the morphological and functional relationship we have just mentioned.

The search for an easy and reliable marker in diagnostic algorithms in acute PTE and DVT has been going on for a long time. D-dimer, a fibrin degradation product, is still considered to be the most suitable biomarker in light of current guidelines. However, the diagnostic value of D-dimer is easily affected by many chronic diseases and cancers. In such cases, a high baseline value leads the patient to overdiagnosis, and many cases of cancer diagnosed with acute PTE have been reported with low D-dimer levels $[5,7,19]$. It has been well documented that some hematological malignancies secrete proteolytic factors. Some patients with hematological malignancies and PTE have normal D-dimer levels because of accelerated degradation of D-dimer. In a large study performed by Qdaisat et al, 1156 cancer patients diagnosed with PTE were retrospectively 
assessed and 35 of those (3.0\%) were found to have normal D-dimer levels [8]. On the other hand, high levels of D-dimer have been reported to be common in cancer patients independent of acute thrombosis. There are even patients diagnosed with cancer when investigating the etiology of high D-dimer level [22]. Contradictory results have been obtained in other studies on this subject. Lee et al. assessed D-dimer levels in 1068 patients suspected with DVT in their retrospective study [23]. While they found similar sensitivity of D-dimer in both patient groups with and without cancer, they observed that negative predictive value was lower in cancer patients. In contrast, in another study performed by Wolde et al., the negative predictive value for D-dimer testing was the same in patients with and without cancer [24]. As a result, the sensitivity and specificity of the D-dimer test in cancer patients are influenced by many clinical variables and thus its reliability varies. This situation requires different clinical evaluations in cancer patients. The mean D-dimer levels were significantly higher in cancer patients; especially in active cancer patients in our study.

Considering the effects of both cancer pathogenesis and chemotherapy on bone marrow, MPV and PDW changes are possible in cancer patients. These morphologic changes also bring about functional changes. Changes in platelet volume and size have functional effects. Compared to small platelets, larger platelets contain more granules. These large granular platelets, which produce larger amounts of prothrombotic factors such as thromboxane A2 and serotonin, rapidly aggregate in the presence of a stimulus to express a greater number of adhesion molecules such as P-selectin and glycoprotein IIb / IIIa [25]. Increased MPV of such platelets with significant changes in size is associated with increased platelet reactivity and shortened bleeding time. In light of all this information, it is reasonable to say that larger platelets carry greater risk for thrombosis and are more active than small platelets. There are some reports about the role of MPV in PTE and cancer prognosis $[12,13]$. However, there is no definite conclusion about the relationship between PTE and MPV in cancer patients. In our study, MPV was found to be significantly higher in cancer cases, even in cases with active cancer. This result supports the association of increased MPV with thrombosis. PDW is accepted as a more specific marker of platelet activation because it does not increase in response to platelet swelling. There are some studies in the literature regarding the relationship of PDW with PTE $[15,26]$. Sevuk et al reported that PDW values were higher in patients who developed acute PTE after DVT than those who did not [27].

Although there are proofs related to alterations of both PDW and MPV in acute PTE, the underlying mechanisms are argumentative. Some investigators have defined the potential mechanism for increased MPV and PDW levels in PTE patients as the increased platelet consumption during the evolution of thrombosis. Large platelets with higher MPV are released from the bone marrow due to thrombopoiesis; stimulated by inflammatory cytokines such as interleukin-1, interleukin-6, and tumor necrosis factor-alpha in acute PTE. Additionally, many other researchers stated that the acute hypoxemia caused by PTE may also be another mechanism for PDW and MPV elevations [27-29]. On the other hand, coexistence of acute PTE and cancer has the potential to affect these increases even more. Our results revealed that despite the low mean of platelet count in patients with active cancer, high mean of MPV and PDW values support this idea. In addition, our results support the hypothesis that the morphological characteristics of the platelets are more determinant than the number of platelets in cancer.

In conclusion, this study demonstrated that active cancer has both a risk factor for PTE and an increase in the severity of the disease. On the other hand, the reliability of the classical diagnostic approach varies in these patients. Our results suggest that D-dimer test has a limited value in the clinical evaluation of acute PTE in cancer patients, and that platelet counts and platelet indexes such as MPV and PDW which can be easily obtained from CBC analysis may be useful in the prediction, diagnosis, and follow-up of PTE. The retrospective design of our study is the limitation of the current investigation. The issue will become clearer with prospective studies with a large number of cases.

\section{ACKNOWLEDGMENTS}

The authors thank the Chest Diseases clinic staff for their efforts to the patients.

\section{CONFLICT OF INTEREST}

The authors declared no conflict of interest. 


\section{ETHICS APPROVAL}

The study was approved by the local ethical committee.

\section{REFERENCES}

1. Timp JF, Braekkan SK, Versteeg HH, Cannegieter SC. Epidemiology of Cancer-Associated Venous Thrombosis. Blood. 2013;122(10):1712-23. DOI: 10.1182/ blood-2013-04-460121 PMID: 23908465.

2. Wang J-G, Geddings JE, Aleman MM, Cardenas JC, Chantrathammachart P, Williams JC, et al. Tumor-Derived Tissue Factor Activates Coagulation and Enhances Thrombosis in a Mouse Xenograft Model of Human Pancreatic Cancer. Blood. 2012;119(23):5543-52. DOI: 10.1182/blood-2012-01-402156 PMID: 22547577.

3. Meikle CKS, Kelly CA, Garg P, Wuescher LM, Ali RA, Worth RG. Cancer and Thrombosis: the Platelet Perspective. Front Cell Dev Biol. 2017;4(147):1-10. DOI: 10.3389/ fcell.2016.00147 PMID: 28105409.

4. Lee EC, Cameron SJ. Cancer and Thrombotic Risk: the Platelet Paradigm. Front Cardiovasc Med. 2017;4(67):1-6. DOI: $10.3389 /$ fcvm.2017.00067 PMID: 29164134.

5. Righini M, Le Gal G, De Lucia S, Roy PM, Meyer G, Aujesky D, et al. Clinical Usefulness of D-Dimer Testing in Cancer Patients with Suspected Pulmonary Embolism. Thromb Haemost. 2006;95(4):715-9. PMID: 16601844.

6. Han D, o Hartaigh B, Lee JH, Cho IJ, Shim CY, Chang HJ, et al. Impact of D-Dimer for Prediction of Incident Occult Cancer in Patients with Unprovoked Venous Thromboembolism. PLoS One. 2016;11(4):e0153514. DOI: 10.1371/ journal.pone.0153514 PMID: 27073982.

7. Lippi G, Franchini M, Biasiutti C, Dellagiacoma G, Salvagno GL, Guidi GC. Increased D-Dimer Value and Occult Cancer in the Absence of Detectable Thrombosis. Haematologica. 2007;92(4):e53-5. DOI: 10.3324/haematol.12237 PMID: 17562593 .

8. Qdaisat A, Wu CC, Yeung S-CJ. Normal D-Dimer Levels in Cancer Patients with Radiologic Evidence of Pulmonary Embolism. J Thromb Thrombolysis. 2019;48(1):174-9. DOI: 10.1007/s11239-019-01863-4 PMID: 31041652.

9. Smyth SS, McEver RP, Weyrich AS, Morrell CN, Hoffman MR, Arepally GM, et al. Platelet Colloquium Participants: Platelet functions beyond hemostasis. J Thromb Haemost. 2009;7(11):1759-66. DOI: 10.1111/j.15387836.2009.03586.x. PMID: 19691483.

10. Jackson SP. The Growing Complexity of Platelet Aggregation. Blood. 2007;109(12):5087-95. DOI: 10.1182/ blood-2006-12-027698 PMID: 17311994.

11. Bilir C, Engin H, Bilir F. Increased Mean Platelet Volume in Deep Vein Thrombosis Patients with Cancer. J Hematol. 2013;2(2):64-8. DOI: $10.4021 /$ jh85w.

12. Machin SJ, Briggs C. Mean Platelet Volume: a Quick, Easy Determinant of Thrombotic Risk? J Thromb Haemost. 2010;8(1):146-7. DOI: 10.1111/j.1538-7836.2009.03673.x PMID: 19874471 .

13. Moharamzadeh P, Rahmani F, Foroughifar S, Shahsavarinia K. Reliability of Platelet Indices for Diagnosing
Pulmonary Embolism; a Brief Report. Adv J Emerg Med. 2019;3(3):e27. DOI: 10.22114/ajem.v0i0.137 PMID: 31410404.

14. Araz O, Albez FS, Ucar EY, Kerget B, Y1lmaz N, Akgun M. Predictive Value of Mean Platelet Volume for Pulmonary Embolism Recurrence. Lung. 2017;195(4):497-502. DOI: 10.1007/s00408-017-0020-7 PMID: 28620755.

15. Razak A, Binti N, Jones G, Bhandari M, Berndt MC, Metharom P. Cancer-Associated Thrombosis: an Overview of Mechanisms, Risk Factors, and Treatment. Cancers. 2018;10(10):380. DOI: 10.3390/cancers10100380 PMID: 30314362.

16. Khorana AA. Malignancy, Thrombosis and Trousseau: the Case for an Eponym. J Thromb Haemost. 2003;1(12):24635. DOI: 10.1111/j.1538-7836.2003.00501.x PMID: 14675077.

17. Baron JA, Gridley G, Weiderpass E, Nyren O, Linet M. Venous Thromboembolism and Cancer. The Lancet. 1998;351(9109):1077-80. DOI: 10.1016/S01406736(97)10018-6 PMID: 9660575.

18. Mezouar S, Frère C, Darbousset R, Mege D, Crescence L, Dignat-George F, et al. Role of Platelets in Cancer and Cancer-Associated Thrombosis: Experimental and Clinical Evidences. Thromb Res. 2016;139:65-76. DOI: 10.1016/j. thromres.2016.01.006 PMID: 26916298.

19. Seizer P, May AE. Platelets and Matrix Metalloproteinases. Thromb Haemost. 2013;110(11):903-9. DOI: 10.1160/ TH13-02-0113 PMID: 23864155.

20. Gremmel T, Perkmann T, Seidinger D, Koppensteiner R, Panzer S, Kopp CW, et al. Differential Impact of Inflammation on Six Laboratory Assays Measuring Residual Arachidonic Acid-Inducible Platelet Reactivity during Dual Antiplatelet Therapy. J Atheroscler Thromb. 2013;20(7):630-45. DOI: 10.5551/jat.17665 PMID: 23739624.

21. Heinmöller E, Weinel RJ, Heidtmann HH, Salge U, Seitz R, Schmitz I, et al. Studies on Tumor-Cell-Induced Platelet Aggregation in Human Lung Cancer Cell Lines. J Cancer Res Clin Oncol. 1996;122(12):735-44. DOI: 10.1007/ bf01209121 PMID: 8954171.

22. Adam SS, Key NS, Greenberg CS. D-Dimer Antigen: Current Concepts and Future Prospects. Blood. 2009;113(13):2878-87. DOI: 10.1182/ blood-2008-06-165845 PMID: 19008457.

23. Lee AYY, Julian JA, Levine MN, Weitz JI, Kearon C, Wells PS, et al. Clinical Utility of a Rapid Whole-Blood D-Dimer Assay in Patients with Cancer Who Present With Suspected Acute Deep Venous Thrombosis. Ann Intern Med. 1999;131(6):417-23. DOI: 10.7326/0003-4819-1316-199909210-00004 PMID: 10498557.

24. ten Wolde M, Kraaijenhagen RA, Prins MH, Büller HR. The Clinical Usefulness of D-Dimer Testing in Cancer Patients with Suspected Deep Venous Thrombosis. Arch Intern Med. 2002;162(16):1880-4. DOI: 10.1001/ archinte.162.16.1880 PMID: 12196087.

25. Holmes MB, Sobel BE, Howard DB, Schneider DJ. Differences Between Activation Thresholds for Platelet P-Selectin and Glycoprotein IIb-IIIa Expression and Their Clinical Implications. Thromb Res. 1999;95(2):75-82. DOI: 
10.1016/s0049-3848(99)00019-5 PMID: 10418796.

26. Yardan T, Meric M, Kati C, Celenk Y, Atici AG. Mean Platelet Volume and Mean Platelet Volume/Platelet Count Ratio in Risk Stratification of Pulmonary Embolism. Medicina. 2016;52(2):110-5. DOI: 10.1016/j.medici.2016.03.001 PMID: 27170484.

27. Sevuk U, Altindag R, Bahadir MV, Ay N, Demirtas E, Ayaz F. Value of Platelet Indices in Identifying Complete Resolution of Thrombus in Deep Venous Thrombosis Patients. Indian J Hematol Blood Transfus.
2015;31(1):71-6. DOI: 10.1007/s12288-014-0365-5 PMID: 25548449 .

28. Vagdatli E, Gounari E, Lazaridou E, Katsibourlia E, Tsikopoulou F, Labrianou I. Platelet Distribution Width: a Simple, Practical and Specific Marker of Activation of Coagulation. Hippokratia. 2010;14(1):28-32. PMID: 20411056.

29. Gasparyan AY, Ayvazyan L, Mikhailidis DP, Kitas GD. Mean Platelet Volume: a Link between Thrombosis and Inflammation? Curr Pharm Des. 2011;17(1):47-58. DOI: $\underline{10.2174 / 138161211795049804}$ PMID: 21247392. 